\title{
PROYECCIONES DINÁMICAS DE POBLACIONES FEMENINAS POR GRUPOS ETÁREOS
}

\author{
Evelyn Álvarez Sierra y Mayra Alfaro Medinaceli
}

\section{RESUMEN}

En el presente trabajo se propone un modelo matemático dinámico para estimar el comportamiento de poblaciones en el tiempo tomando en cuenta la estructura de edades. Se discretiza la ecuación diferencial en derivadas parciales que se obtiene como modelo, utilizando el Método Numérico de Líneas. Para su desarrollo computacional, se utilizó el código DASSL y datos de los censos de Bolivia de 1992 y 2001, se comparó los resultados obtenidos numéricamente con los datos reales. Con el modelo propuesto, se realizaron proyecciones del comportamiento de la población femenina; se observó una tendencia al crecimiento en general y, en particular, la estabilidad en la cantidad de individuos con edades entre $70 \mathrm{y}$ 75 años.

Palabras Clave: Poblaciones, Ecuaciones Diferenciales Algebraicas, Modelos Matemáticos, Método Numérico de Líneas. 